\section{Index des notions}

adresse

- interdiscursive $163,166,595,611$

- termes de 116-117, 134, 468

allusion $85,143,153,170,186,217,298$, $302,347-348,427-429,436,438$, 440-441, 443, 445, 448-449, 451-453, $458,559,571,612$

ambiguïté $46,76,84,87,111,113,250,257$, $285,299,346,355-356,564$

anaphore, cataphore 45, 93-94, 131, 205, 207-208, 261, 281, 290, 317

ancrage énonciatif

- couche primaire vs secondaire XXV, 102, 109-110, 113-115, 117, 130-136, 139, 144-145, 338

- versant référentiel vs modal XXV, 59, $107,109,120,130,134-136,139-140$, 151, 281, 321, 329, 356

- divisé, partagé (bivocal-DIL) 59, 117, 129-134, 136, 138, 142, 144, 151, 316, 321, 338, 340, 345

- double hiérarchisé (DD) XXVI, 118, 122, 126-128, 142, 144, 151, 233, 285, 298, 306, 338, 345

- unifié, intégré (DI, MAE, MAS) XXVI, 102-103, 106, 108, 114, 140, 144, 151, $217,243,285,303,305,338-340$, 345-346, 356

apposition 121, 131, 205, 257, 266, 317 appropriation $383,462,506-509,514$, $518-520,522,539,553-554$ argumentation XXVI, 40, 84-85, 87, 208, $210,213-215,335,355,433,478,481$, 578

articulation énonciative XVIII, XXV, 35, 38, 70, 102, 106, 129, 131, 133, 144-146, $186,201,277,328,336,366,423$, 476

assimilation/dissimilation (fonction dissimilatrice de la RDA) XXVII, 378, 458-463, 466, 475, 483, 491, 502, 505, 522, 529, 534, 543, 546, 550-551, 554, 598 auto-représentation (ARD) XXV, 9, 11-12, $16-17,19,21-22,24,28-29-35,89$,
$102,154,176,204,246,249,251,264$, 286, 289, 472-475, 477

autonymie: 122-123, 126, 199-201, 211-212, 215, 220, 231, 246-324, 329, 335, 339, 352, 360-361, 364-365, 625

- blocage de synonymie $254-255,264$. 287-288, 337

- conceptions diverses 250-251

- construit vs non construit 259-261

- dire vs montrer 251, 261-262

- iconicité 255-256, 261

- recatégorisation nominale 256-259, 321

- autonymie et « fidélité »/

paraphrase $169-170,181,231,240$,

267, 272, 243, 332, 334, 584

- référence et sens du $\boldsymbol{e}$ autonyme 252 , $255,263-265,271,278-285$

- autonymie comme masque 190-194, 282-284

- voir aussi discours direct, bivocal, modalisation autonymique

Autre du langage vs autres discours XXVIII, $378,383,416,500,506,509-511,518$, 527, 546, 549-550, 552, 563, 565, 567, $569,573,577,582$

barrière de contact XXVIII, 499-500, 561, 563 bascule (de mode vs hybridité) 110, 116, 305, 356 bêtise

- voir aussi doxa 512, 577, 598, 613, 618

- voir aussi imaginaire 548, 566, 569

bivocal-DIL 40, 42, 45, 56-59, 100, 109, 113, 117, 129-146, 205-209, 217-218, 231, 246-247, 265, 277, 301, 310-324, $337-351,355,357,384,428-429,446$, 572,625

- « effet de DIL » 115

bord, bordure XXVIII, XXIX, 378, 494, 502, 543-544, 546, 550, 553, 555-561, 563, 566-583, 592-593, 595-598, 600, 604, 607-608, 622, 625-631

- bord de la bordure 
- bord dans la bordure

voir aussi limite, frontière, tracé

canal

- oral/écrit $17,19,22,39,42-43,52$, 123-124, 157-158, 164, 211, 213, 221,

272, 308, 345, 348, 353, 361, 447, 481,

$584,587,595$

- support $47-48,52,54-55,157,345$, 622

catégorisation XXV, 7, 13, 42, 54-55, 58, 66, 79, 88, 102-103, 108, 168-170, 176, 199-225, 242, 354, 375, 585, 617

voir aussi Discours indirect

citation 7, 13-14, 19-20, 114, 120,143, 162-163, 180-181, 185, 190, 201, 251, 260, 268, 273, 275-276, 296, 331, 360-371, 437, 441, 447, 477-478, 481, 486, 490, 507, 513-514, 517, 527-528, $533,549,553,558,564-567,569-570$, $574,578,588,595,597-598,605,612$, 624,631

- citationnel 370-371, 528, 564-566, 569,575

coïncidence/non coïncidence référentielle

A/a XV, 8-9, 17, 21-22, 25, 28-29, 275, 292-293, 295-296, 302, 376-377, 391, 414, 425, 431, 451, 473, 548, 554

voir aussi non-coïncidences du dire concordance /discordance inter/intradiscursive $59,116,133,153,329,347$, 429-431, 438-443, 453, 461-463, 496, 521, 538

condition pour RDA

- condition de non-performativité ; 17 , 21, 30, 204

- condition de représentativité, 12 , $54-55,340$

configuration (fonction configurative, auto-configurante) VIII, XIX, XXVI, 377-378, 422, 426, 451, 463, 466, 471, 480, 484-485, 487, 490-491, 511, 546, 550-552, 555, 557-559, 563-566, 568, 571,573

conscience $9,377,383-385,391,400,422$ - degré de 427, 450, 453, 457-458, 461, 463-465, 469, 482-484, 548, 565 contacts

- entre modes 354-355

voir aussi ambiguïté, indécidabilté, neutralisation

- RDA comme contact $378,477,488$, $556-557,559,568-569,572,580,625$

voir aussi barrière, interface

continuum vs distinctivité XIV, XXIII, XXIV, 45-46, 64, 86, 284-285, 331-333, 335, 338-339

voir aussi Formule, hybridité-mixité, langue/ discours

co-référence 97, 119, 208, 256, 290, 350, 355 contexte (recontextualisation) XI, XV, XXII, XXIV, XXV, 14, 26, 35-36, 38, 42, 44, $46,53,62,83-85,120,132,148-152$, 154-160, 230-232, 234-235, 238, 241, $438,452,460,537,539,610$

- contexte d'accueil 120, 172-175, 438, $460,537,539$

- contexte représenté 155-172, 610

- co-texte (voir intradiscours) 55, 108, 118, 120-121, 142, 152-153, 155, 163-166, 174, 178-182, 184, 186, 190, $263,280,285,329,430,438,444$

- double recontextualisation 69,147 , 154-155, 176-185

conversion (métadiscursive) XXVIII, 51, 107, 114, 120,173, 257, 261, 285, 320, 378, 453, 519-520, 533, 546, 549-550, 553, 561

cumul

- de modes de RDA XXVI, 97, 241, 299-300, 305, 346, 356

- usage et mention 249, 284, 286-287, 289-290, 294, 313, 364

dedans-dehors (intérieur-extérieur) XXVIII, 153, 376, 392, 395, 490, 492-496, 498-503, 505, 508, 513, 519-520, 522-526, 532, 542-546, 548, 550,551, 556-559, 561-563, 566, 568-569, 571-572, 574, 582, 598, 607, 626-627 - géographie vs topologie XXVIII, 295, 378, 407, 457, 547, 550-551, 574 voir aussi conversion métadiscursive descriptions définies 136-138, 156, 233 
dialogisme XVI, XVIII, 20, 64-65, 101, 132, 296, 375, 377, 379, 383-393, 396-397, 399-400, 413, 417-419, 433-435, 445, 457, 463, 521, 624

- auto-dialogisme 9, 291, 296

- dialogisme interlocutif 291-292, 383, 391

voir aussi indice, marqueur

diffraction, spectrographie $35,52-53,55$, 107, 311

discordance énonciative voir bivocal discours autre

- attaché/associé 171, 185, 304, 429, 568, 577, 581-582, 588, 593, 597, 599, 602, 604, 607-610, 612, 622-623, 626, 629

- conjoint 71, 87, 91-95, 100

- de soi vs d'autrui (voir ARD/RDA) XIV, 18-19, 24, 39, 66, 264, 272, 453,

472-474

- factuel vs virtuel $21,39-40,52$, 60-61, 269-270, 476-477, 574

- intérieur 43-46, 74, 311

- objet vs source XXV, XXVI, 32-33, 53, $65,69,101,335-337,340,352,587$

discours direct (DD)

- accès à la référence 118-127

- vs en direct $37,106,120,127,179$, 190, 284, 516

- énoncé par L 123-125

voir aussi ancrage énonciatif, autonymie, embrayage, Formule de mode, littéralité, fidélité, hybridité/mixité, récursivité, référence

discours indirect (DI) :

- DI et catégorisation 205, 217-220

- narrativisé 54, 61, 217, 220-221, 243 ,

340-341, 357

- « règles de transposition " XIV, XV, XXII, 104-107, 111, 126, 139, 202, 229-232, 237, 240-241

voir aussi ancrages énonciatifs, cumul, Formule de mode, extension extraphrastique, mixité, récursivité, thème

discours indirect libre (DIL) voir bivocal discordance (voir concordance) disjonction (plan de clivage entre modes) 56-59, 114, 126, 266, 276

dissimilation (voir assimilation)

-tracé dissimilateur (configurateur...) XXVII, 10-11, 359, 378, 424, 426, 437, 449, 451-452, 463-464, 466, 502, 551-553, $556,558-559,561,565,568$

doxa, idées reçues $513,526,540-541,548$, $570,578,601,604,612,621-622$

embrayé (désembrayé, désactivé) 104, 119-122, 127, 277

voir aussi autonymie comme masque enchaînement (de formes de RDA) XXVI, 218, 240, 346, 354, 357-359

entrée dans le langage 504, 506, 508-511, 515, 518, 520

voir aussi greffe, infans

étagement, surplomb métadiscursifs XVIII, XXIX, 4-5, 7-8, 10, 34, 123, 179, 339, 447, 461, 473, 484, 490, 499, 534, $550-551,554,558,575,593$

ethos voir identité

évidentiel, médiatif 76-79, 97

voir aussi MAS

expressif (élément) 52, 107-108, 112-113, $116,131,134,140$

voir aussi ancrage modal secondaire extension extraphrastique du $\boldsymbol{e} 141$, 218-219, 348-349, 427, 438, 560

extériorité discursive, langagière $\mathrm{XIX}, \mathrm{XXVI}$, XXVIII, XXIX, 101, 153, 161, 359, 375-376, 386, 392, 398-402, 413, 422, 428, 432, 434, 441, 457-458, 461, 483-485, 490, 506, 515, 517-519, 524, 532, 535, 543-552, 557-559, 570, 574, 625 - extériorité interne 101, 377, 379, 384, 386, 393, 395, 397, 399-400, 418, 451, 458, 463, 482, 504, 519, 522, 524, 532, $543,550,552$

voir aussi hétérogénéité constitutive, dedans-dehors, frontière absente

famille (de modes de RDA) XXVI, 59, 345-346, 356, 361

fidélité (textuelle, littérale) XIV, XVIII, 24, 36, $69,81,124-126,150,169,171,179,181$, 
199, 230-231 241, 242, 245, 247, 261, 263, 265-266, 269-272-277, 279, 284, 298-300, 302-304, 306-309, 311-312, $314-316,323,330-332,334,339,356$, 361-362, 370

- degré de 266-270, 316, 361

voir aussi autonymie, hybridité, îlot textuel formes (de RDA, voir zones de)

- formes complexes (voir cumul, enchaînement)

formule (de mode) XXV, XXVI, 327, 338-344, 347-348, 359, 558, 580

frontière XXVII,

- entre zones de méta-discours 4,10, 12-13, 17, 25, 29, 54, 227, 364, 437, 565 - de phrase, d'énoncé (voir marquage extension) 141, 349, 353

- dans le discours avec les autres et l'Autre 359, 442, 447, 449-450, 464-466, 490-493, 495-496, 498, 500-503, 532, 543-544, 550-553, 555-557, 563-565, 567-568, 571, 573

- absente 402, 550, 553, 564

- zone (interface) XXVIII, 378, 498, 500-503

voir dedans-dehors, continuum

générique XIV, 46, 162, 185-186, 192, 236, 238, 268, 461, 468, 470, 480, 536, $569,574-575,577,582,584-585,588$, 593-594, 598-599, 605, 607, 612-613, 626,630

voir index des genres géographie dedans/dehors XXVIII, 295, 378, 407, 457, 547, 550-551, 574

voir aussi extériorité interne

greffe (dénaturation, prothèse d'origine, du langage) XXVIII, 504-507, 509, 513, 518, 543,554

hétérogénéité (constitutive/représentée) $X V$, XVIII, XXVII, XXIX, 8-9, 40, 64, 101, 295-296, 336, 371, 375-377, 380, 387, 389, 393-395, 397, 400-402, 404, 412-413, 416-418, 420-421, 426, 430, 451-452, 482-483, 489, 491, 521, 569, 571-572, 599 homomorphie A/a 35-36, 102, 194, 265, 370

- recontextualisation

homomorphe 190-192

hybridité (mixité) XXIV, 64, 139, 141, 305, 309, 331-334, 356, 384

- DD non textuel,

paraphrastique 169-170,181, 240, 267, $272,243,332,334,584$

- DD avec que 306, 309, 332, 356

- DI sans que 141, 341, 349, 355

- quasi-textuel (DI, MAS) 242, 245, 303-304, 311

voir aussi bascule, bivocal, continuum

identité

- production, construction, indicateur, marqueur... XXVIII, 464-473, 476, 479-480, 486, 490, 496-497, 520, 525, $534-535,550,556,561,568,573$

- défaut, perte, vacillement... 501-502, $529,531,542,572,578$

îlot textuel 81, 245, 303-307, 361

voir, MAE, îlot textuel vs DD

image

- vs acte 35-36, 38, 55-59, 123,

125-126, 163-164, 168-169, 172, 200,

204, 232, 238, 240, 246, 264-272,

274-277, 298, 312, 314-315, 377,

- de soi XXVI, XXVII, XXIX, 378, 422, 424, 426, 465-473, 475-476, 479-481, 484, 486-488, 490-497, 500-501, 503, $546,549,551,554-555,560-561,563$, 573,630

imaginaire XXVII, XXVIII, 10, 15, 171, 378, 403, 407, 410-412, 414-416, 421, 451, 464, 467, 490-491, 496, 500, 502, $504,509,515,532-533,544,546-549$, $552,-554,561,563-564,566$

inappartenance du langage XXVIII, 371, 378, 492, 514, 520, 564, 566, 569

incise XXVI, 22, 24, 101, 131, 141, 143, 167, 205-207, 261, 309, 316, 332, 346, 351-353

indécidabilité 101, 299, 346, 355-356, 426, 464

indice (vs marque, trace) 167, 351-353, 359, 391, 428-436, 438-443, 445-446, 468 
infans 490, 492, 494-496, 498, 504-505, 508,518

voir langage (entrée dans)

injure, insulte 108-109, 116, 134, 200, 204, 525

interface XXVIII, 378, 498-499, 501-502, 558, 561-563, 582, 592-593, 597, 599, 605-607, 625-628

interlocution (interlocuteur, interlocutif) 20, 103, 106, 116, 132, 134, 156, 193 , 204, 283, 324, 382, 391, 396, 427, 440, 443, 449-450, 467, 476, 484, 559

voir aussi dialogisme, non-coïncidences du dire

introducteur 81, 122, 166-168, 202, 205 , 208-209, 211-212, 260, 306, 349, 354, 358

voir aussi recteur

- vs annonceur XXI, 131, 143, 167, 205-207, 218, 243, 260, 317, 319-320, $329,354,358,608$

ironie $159,173,440,446,571-572,577$

irreprésentable XIX, 194, 375-376, 396, 413, $416,500,547,549-550,553,561$

jurons 108-109, 112, 114, 116, 134, 200

langue vs discours XVII, XIX-XXVIII, 10, 16, $38,56,59,66,68-69,83,86,120$, 129, 131-132, 147-151, 154, 201, 204, 226-228, 236, 251-253, 255, 258-259, 263-265, 272, 274, 277, 279-280, 282-285, 293-295, 327-359, 375-376, 395-397, 399, 406, 412-413, 419, 430, 422-436, 506, 510, 514, 518-521, 526, $529,545,554,580,625$

limite XXVIII, 465, 492-493, 495-496, 498, 500-503, 511, 529, 532, 543-544, 550, 552, 560-561, 565, 567 - délimiter 450, 495, 550, 557, 563, 567, 569,572

- hors limites, illimité $371,376,422$, 457, 572

marque 167, 256-257, 347-349, 351-354, 422, 426, 430-434, 438, 443
- degré de marquage 55, 109, 118, 142-143, 153-156, 186, 217-218, 261, 283, 293, 298-302, 307-308, 341, 348, 428-429, 438, 441-442, 447, 452-453, 457, 459, 463, 474, 528, 558, 571-572, $585,588-589,594,608,628$

- marquage rétroactif 359,528

- marqueur dialogique $435,438,448$, 451

- zéro voir allusion

voir aussi indice, trace, langue vs discours MAS (modalisation en assertion seconde) 33 , 40, 71-75, 79, 82-97, 100-101, 108, 143, $322,340,614$

méconnaissance XXIII, XXVIII, 9, 406, 412, $482,484,533,564,566$

- fonction de, force de $410,532,547$, 550

voir imaginaire

mémoire (inter/intradiscursive) XXVII, 151, $363,381,390,394,402,412-413,433$, 438, 451, 488-489, 574, 588, 615, 624 - domaine, espace, réseau ; 152 , 394-395, 398, 401-402, 413, 422, 427, 489

- différence, partage, rencontre, extension 170, 180, 186, 401, 427-429, 431, 436, 441-447, 451, 453-454, 463, $483,537-538,623,629$

mixité voir hybridité modalité autonymique (MA) XV, 23, 246-249, 285-286-297, 307-310

voir non-coïncidences du dire modalité autonymique d'emprunt (MAE) 16, 71, 81, 96, 117, 169, 231, 242, 247, 249, 271, 288-289, 297-303, 307-310, 539, $541,543,559,588-589$

- MAE vs DD 285, 297-299, 308-311-312

- MAE « paraphrastique » 231

voir aussi allusion monologique (discours) 385-386, 422, 465, $489,569,571,592,595-596$

narrativisé voir DI neutralisation XXVI, 101, 167, 238, 258, 272, 299, 346, 351-352, 355, 443, 615 
non-coïncidences du dire XV, 8-9, 292-293, 295-296, 302, 377, 391, 414-415, 431, 451, 473, 548, 554

parole propre (« de soi ») XXVIII, 18, 378, 426, 450, 452, 490-492, 495, 503-504, $508,510,517,519-520,522,526-528$, 531-534, 536, 538, 541, 543-544, 546-547, 549-553, 557-558-561, 563, 570

vs parole imposée, " comme citée ", automatisme mental, « voix » 501, 522-529, 570

voir aussi appropriation

performativité,: XX, 11, 18, 20-23, 26, 30-31, $176,204,458,460,473,488,592-593$

polyphonie (conception de l'énon-

ciation) $63-64,84-85,386-387,391$, 417-420, 434

pondération énonciative $A / a$ (en DI et bivocal) $115,134-138,146$ positionnement $378,449,466-471$, 474-477, 480, 486-487, 552-553

prélèvement méta-discursif XIX, XXVII, 155, $163,173,178,363,378,422-464,552$

- complémentaire 426, 458, 489, 491, 551-552, 557

présence $v s$ représentation (de l'altérité discursive) XVI, XXVII, 3, 64, 375-378, 404, 422-426, 428, 442, 449-453, 458, $461,463,466,472,475-476,537,550$, $555,561,563-564$

présentation de soi $466-467,470-471,475$, 479-480, 486, 630 réception (diathèse, pôles de) 9, 20, 43, 76, 156-158, 161, 176, 180, 211-213, 238, 270, 291, 329, 443, 451, 456, 463, 489, 545,560

- (représenté comme) reçu vs énoncé $32,51,96,188,211,442,444$, 447, 457, 522, 537, 545, 559, 581, 591

recteur, rection $131,142-143,167,202$, 205-207, 329, 354, 358

récursivité $90,127-129,357$

référence $X I, 5-6,10-13,16-18,21-22$, $25,28,35-36,38-40,42,46-47,49$, $53-54,65,72,75-76,80,118-127$, 137-138, 147, 178, 180, 184, 194, 204, 247-249, 251-252, 262, 288-290, 297 voir aussi ancrages énonciatifs, autonymie, co-référence, discours direct, fidélité réflexivité XVIII, 4-5, 8-9, 11, 34, 64-65, 99, 248-249, 280, 307, 551, 554

stéréotype (cliché, formule, modèle, patron, routine) $42,60,213,349,357,362$, 390, 435, 457-458-461, 479, 485, 488, $490,575,593$

thème (du DA représenté) 54, 201, 242-245, 610

trace $186,397-399,402-404,422,428$, 430, 434, 449

voir aussi interdiscours, présence type, token XXV, 11-12, 120, 201, 204, 227, 247, 251-255, 257-259, 263-264, 274-275, 278-280, 283, 307, 364

Zone de formes de RDA XXVI, 327, 338-344, 347-348, 359, 558, 625 